\title{
A Tough Egg to Crack
}

The approach to clinical conundrums by an expert clinician is revealed through the presentation of an actual patient's case in an approach typical of a morning report. Similar to patient care, sequential pieces of information are provided to the clinician, who is unfamiliar with the case. The focus is on the thought processes of both the clinical team caring for the patient and the discussant.

This icon represents the patient's case. Each paragraph that follows represents the discussant's thoughts.

Rabih M. Geha, MD ${ }^{1,2 *}$, Gurpreet Dhaliwal, MD1,2, Marion G. Peters, MD,1 Reza Manesh, MD³

${ }^{1}$ Department of Medicine, University of California San Francisco, San Francisco, California; ${ }^{2}$ Medical Service, San Francisco VA Medical Center, San Francisco, California; ${ }^{3}$ Department of Medicine, Johns Hopkins Hospital and Johns Hopkins University School of Medicine, Baltimore, Maryland.

A 68-year-old woman presented to the emergency department with altered mental status. On the morning prior to admission, she was fully alert and oriented. Over the course of the day, she became more confused and somnolent, and by the evening, she was unarousable to voice. She had not fallen and had no head trauma.

Altered mental status may arise from metabolic (eg, hyponatremia), infectious (eg, urinary tract infection), structural (eg, subdural hematoma), or toxin-related (eg, adverse medication effect) processes. Any of these categories of encephalopathy can develop gradually over the course of a day.

One year prior, the patient was admitted for a similar episode of altered mental status. Asterixis and elevated transaminases prompted an abdominal ultrasound, which revealed a nodular liver and ascites. Paracentesis revealed a high serum-ascites albumin gradient. The diagnosis of cirrhosis was made based on these findings. Testing for viral hepatitis, autoimmune hepatitis, hemochromatosis, and Wilson's disease were negative. Although steatosis was not detected on ultrasound, nonalcoholic fatty liver disease (NAFLD) was suspected based on the patient's risk factors of hypertension and type 2 diabetes mellitus. She had four additional presentations of altered mental status with asterixis; each episode resolved with lactulose.

Other medical history included end-stage renal disease (ESRD) requiring hemodialysis. Her medications were labetalol, amlodipine, insulin, propranolol, lactulose, and rifaximin. She was originally from China and moved to the United States 10 years earlier. Given concerns about her

*Address for correspondence: Rabih M. Geha, MD, San Francisco VA Medical Center, 4150 Clement St (111), San Francisco, CA 94121, Telephone: 415-2212810; Fax: 415-379-9669; E-mail: rabih.geha@ucsf.edu

Received: December 19, 2017; Revised: May 3, 2018; Accepted: May 7, 2018

๑ 2018 Society of Hospital Medicine DOI 10.12788/jhm.3063 ability to consistently take medications, she had moved to a long-term facility. She did not use alcohol, tobacco, or illicit substances.

The normalization of the patient's mental status after lactulose treatment, especially in the context of recurrent episodes, is characteristic of hepatic encephalopathy, in which ammonia and other substances bypass hepatic metabolism and impair cerebral function. Hepatic encephalopathy is the most common cause of lactulose-responsive encephalopathy, and may recur in the setting of infection or nonadherence with lactulose and rifaximin. Other causes of lactulose-responsive encephalopathy include hyperammonemia caused by urease-producing bacterial infection (eg, Proteus), valproic acid toxicity, and urea cycle abnormalities.

Other causes of confusion with a self-limited course should be considered for the current episode. A postictal state is possible, but convulsions were not reported. The patient is at risk of hypoglycemia from insulin use and impaired gluconeogenesis due to cirrhosis and ESRD, but low blood sugar would have likely been detected at the time of hospitalization. Finally, she might have experienced episodic encephalopathy from ingestion of unreported medications or toxins, whose effects may have resolved with abstinence during hospitalization.

The patient's temperature was $37.8^{\circ} \mathrm{C}$, pulse 73 beats/ minute, blood pressure $133 / 69 \mathrm{mmHg}$, respiratory rate 12 breaths/minute, and oxygen saturation $98 \%$ on ambient air. Her body mass index (BMI) was $19 \mathrm{~kg} / \mathrm{m}^{2}$. She was somnolent but was moving all four extremities spontaneously. Her pupils were symmetric and reactive. There was no facial asymmetry. Biceps and patellar reflexes were 2+ bilaterally. Babinski sign was absent bilaterally. The patient could not cooperate with the assessment for asterixis. Her sclerae were anicteric. The jugular venous pressure was estimated at 13 $\mathrm{cm}$ of water. Her heart was regular with no murmurs. Her lungs were clear. She had a distended, nontender abdomen with caput medusae. She had symmetric pitting edema in her lower extremities up to the shins. 
The elevated jugular venous pressure, lower extremity edema, and distended abdomen suggest volume overload. Jugular venous distention with clear lungs is characteristic of right ventricular failure from pulmonary hypertension, right ventricular myocardial infarction, tricuspid regurgitation, or constrictive pericarditis. However, chronic biventricular heart failure often presents in this manner and is more common than the aforementioned conditions. ESRD and cirrhosis may be contributing to the hypervolemia.

Although Asian patients may exhibit metabolic syndrome and NAFLD at a lower BMI than non-Asians, her BMI is uncharacteristically low for NAFLD, especially given the increased weight expected from volume overload. There are no signs of infection to account for worsening of hepatic encephalopathy.

Laboratory tests demonstrated a white blood cell count of $4400 / \mu \mathrm{L}$ with a normal differential, hemoglobin of $10.3 \mathrm{~g} / \mathrm{dL}$, and platelet count of 108,000 per cubic millimeter. Mean corpuscular volume was $103 \mathrm{fL}$. Basic metabolic panel was normal with the exception of blood urea nitrogen of 46 $\mathrm{mg} / \mathrm{dL}$ and a creatinine of $6.4 \mathrm{mg} / \mathrm{dL}$. Aspartate aminotransferase was 34 units $/ L$, alanine aminotransferase 34 units/L, alkaline phosphatase 289 units/L (normal, 31-95), gamma-glutamyl transferase 104 units (GGT, normal, 12-43), total bilirubin $0.8 \mathrm{mg} / \mathrm{dL}$, and albumin $2.5 \mathrm{~g} / \mathrm{dL}$ (normal, 3.5-4.5). Probrain natriuretic peptide was $1429 \mathrm{pg} / \mathrm{mL}$ (normal, <100). The international normalized ratio (INR) was 1.0. Urinalysis showed trace proteinuria. The chest $x$-ray was normal. A noncontrast computed tomography (CT) of the head demonstrated no intracranial pathology. An abdominal ultrasound revealed a normal-sized nodular liver, a nonocclusive portal vein thrombus (PVT), splenomegaly (15 cm in length), and trace ascites. There was no biliary dilation, hepatic steatosis, or hepatic mass.

The evolving data set presents a mixed picture about the state of the liver. The distended abdominal wall veins, thrombocytopenia, and splenomegaly are commonly observed in advanced cirrhosis, but these findings reflect the associated portal hypertension and not the liver disease itself. The normal bilirubin and INR suggest preserved liver function and decrease the likelihood of cirrhosis being responsible for the portal hypertension. However, the elevated alkaline phosphatase and GGT levels suggest an infiltrative liver disease, such as lymphoma, sarcoidosis, or amyloidosis.

Furthermore, while a nodular liver on imaging is consistent with cirrhosis, no steatosis was noted to support the presumed diagnosis of NAFLD. One explanation for this discrepancy is that fatty infiltration may be absent when NAFLD-associated cirrhosis develops. In summary, there is evidence of liver disease, and there is evidence of portal hypertension, but there is no evidence of liver parenchymal failure. The key features of the latter - spider angiomata, palmar erythema, hyperbilirubinemia, and coagulopathy - are absent.

Noncirrhotic portal hypertension (NCPH) is an alternative explanation for the patient's findings. NCPH is an elevation in

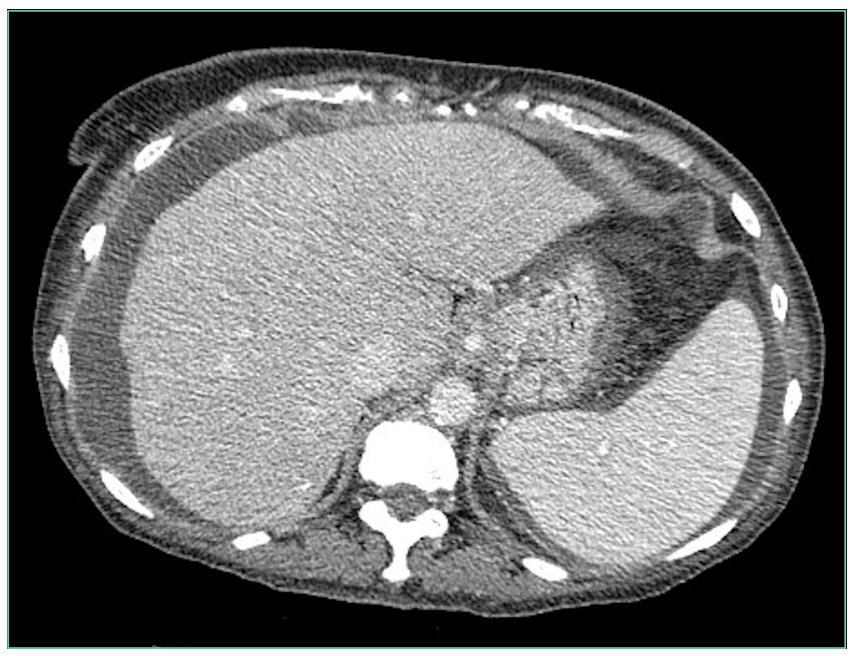

FIG 1. CT of the abdomen and pelvis with contrast demonstrating a nodular liver, ascites, and splenomegaly

the portal venous system pressure that arises from intrahepatic (but noncirrhotic) disease or from extrahepatic disease. Hepatic schistosomiasis is an example of intrahepatic but noncirrhotic portal hypertension. PVT that arises on account of a hypercoagulable condition (eg, abdominal malignancy, pancreatitis, or myeloproliferative disorders) is a prototype of extrahepatic $\mathrm{NCPH}$. At this point, it is impossible to know if the PVT is a complication of NCPH or a cause of NCPH. PVT as a complication of cirrhosis is less likely.

An abdominal CT scan would better assess the hepatic parenchyma and exclude abdominal malignancies such as pancreatic adenocarcinoma. An echocardiogram is indicated to evaluate the cause of the elevated jugular venous pressure. A liver biopsy and measurement of portal venous pressure would help distinguish between cirrhotic and noncirrhotic portal hypertension.

Hepatitis A, B, and C serologies were negative as were antinuclear and antimitochondrial antibodies. Ferritin and ceruloplasmin levels were normal. A CT scan of the abdomen with contrast demonstrated a nodular liver contour, splenomegaly, and a nonocclusive PVT (Figure 1). A transthoracic echocardiogram showed normal biventricular systolic function and size, normal diastolic function, a pulmonary artery systolic pressure of $57 \mathrm{mmHg}$ (normal, < 25), moderate tricuspid regurgitation, and no pericardial effusion or thickening. The patient's confusion and somnolence resolved after two days of lactulose therapy. She denied the use of other medications, supplements, or herbs.

Pulmonary hypertension is usually a consequence of cardiopulmonary disease, but there is no exam or imaging evidence for left ventricular failure, mitral stenosis, obstructive lung disease, or interstitial lung disease. Portopulmonary hypertension (a form of pulmonary hypertension) can develop as a consequence of end-stage liver disease. The most common cause of hepatic encephalopathy due to portosystemic shunting is 
cirrhosis, but such shunting also arises in $\mathrm{NCPH}$.

Schistosomiasis is the most common cause of NCPH worldwide. Parasite eggs trapped within the terminal portal venules cause inflammation, leading to fibrosis and intrahepatic portal hypertension. The liver becomes nodular on account of these changes, but the overall hepatic function is typically preserved. Portal hypertension, variceal bleeding, and pulmonary hypertension are common complications. The latter can arise from portosystemic shunting, which leads to embolization of schistosome eggs into the pulmonary circulation, where a granulomatous reaction ensues.

A percutaneous liver biopsy showed granulomatous inflammation and dilated portal venules consistent with increased resistance to venous inflow (Figure 2). There was no sinusoidal congestion to indicate impaired hepatic venous outflow. Mild sinusoidal and portal fibrosis and increased iron in Kupffer cells were noted. There was no evidence of cirrhosis or steatohepatitis. Stains for acid-fast bacilli and fungi were negative. 16S rDNA (a test assessing for bacterial DNA) and Mycobacterium tuberculosis polymerase chain reactions were negative. The biopsy confirmed the diagnosis of noncirrhotic portal hypertension.

Hepatic granulomas can arise from infectious, immunologic, toxic, and malignant diseases. In the United States, immunologic disorders, such as sarcoidosis and primary biliary cholangitis, are the most common causes of granulomatous hepatitis. The patient lacks extrahepatic features of the former. The absence of bile duct injury and negative antimitochondrial antibody exclude the latter. None of the listed medications are commonly associated with hepatic granulomas. The ultrasound, CT scan, and biopsy did not reveal a granulomatous malignancy such as lymphoma.

Infections, such as brucellosis, $\mathrm{Q}$ fever, and tuberculosis, are common causes of granulomatous hepatitis in the developing world. Tuberculosis is prevalent in China, but the test results do not support tuberculosis as a unifying diagnosis.

Schistosomiasis accounts for the major clinical features (portal and pulmonary hypertension and preserved liver function) and hepatic pathology (ie, portal venous fibrosis with granulomatous inflammation) in this case and is prevalent in China, where the patient emigrated from. The biopsy specimen should be re-examined for schistosome eggs and serologic tests for schistosomiasis pursued.

Antibodies to human immunodeficiency virus, Brucella,

Bartonella quintana, Bartonella henselae, Coxiella burnetii, Francisella tularensis, and Histoplasma were negative. Cryptococcal antigen and rapid plasma reagin were negative. IgG antibodies to Schistosoma were 0.21 units (normal, < 0.19 units). Based on the patient's epidemiology, biopsy findings, and serology results, hepatic schistosomiasis was diagnosed. Praziquantel was prescribed. She continues to receive daily lactulose and rifaximin and has not had any episodes of encephalopathy in the year after discharge.

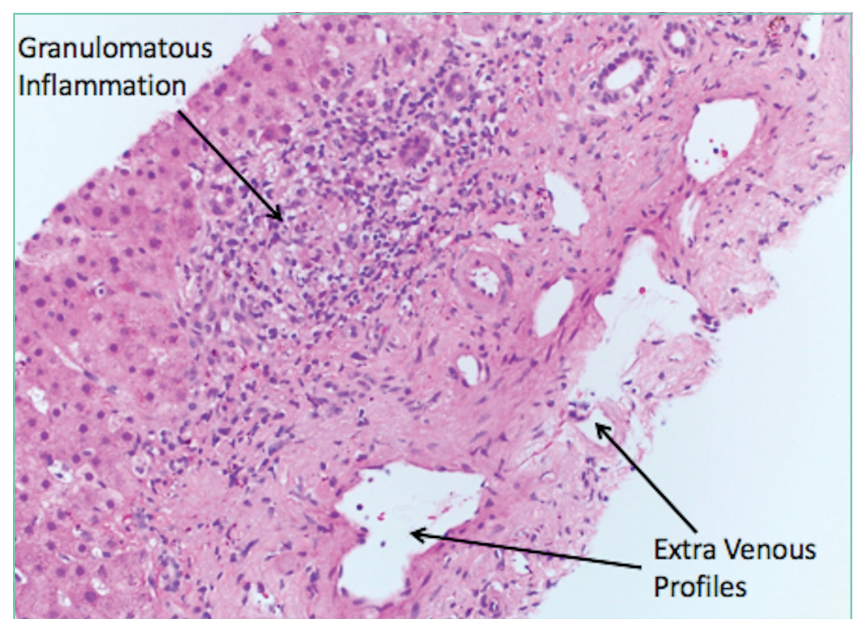

FIG 2. Liver biopsy specimen demonstrating granulomatous inflammation and dilated portal venules in the absence of hepatic congestion suggests an intrahepatic portal venous inflow disorder.

\section{COMMENTARY}

Portal hypertension arises when there is resistance to flow in the portal venous system. It is defined as a pressure gradient greater than $5 \mathrm{mmHg}$ between the portal vein and the intra-abdominal portion of the inferior vena cava. ${ }^{1}$ Clinicians are familiar with the manifestations of portal hypertension - portosystemic shunting leading to encephalopathy and variceal hemorrhage, ascites, and splenomegaly with thrombocytopenia - because of their close association with cirrhosis. In developed countries, cirrhosis accounts for over $90 \%$ of cases of portal hypertension. ${ }^{1}$ In the remaining $10 \%$, conditions such as portal vein thrombosis primarily affect the portal vasculature and increase resistance to portal blood flow while leaving hepatic synthetic function relatively spared (Figure 3). Therefore, cirrhosis cannot be inferred with certainty from signs of portal hypertension alone.

Liver biopsy is the gold standard for the diagnosis of cirrhosis, but this method is increasingly being replaced by noninvasive assessments of liver fibrosis, including imaging and scoring systems. ${ }^{2}$ Clinicians often infer cirrhosis from the combination of a known cause of liver injury, abnormal liver biochemical tests, evidence of liver dysfunction, and signs of portal hypertension. ${ }^{3}$ However, when signs of portal hypertension are present, but liver dysfunction cannot be established on physical exam (eg, palmar erythema, spider nevi, gynecomastia, and testicular atrophy) or laboratory testing (eg, low albumin, elevated INR, and elevated bilirubin), noncirrhotic causes of portal hypertension should be considered. In this case, the biopsy showed vascular changes that suggested impaired venous inflow without bridging fibrosis, which pointed to $\mathrm{NCPH}$.

$\mathrm{NCPH}$ is categorized based on the location of resistance to blood flow: prehepatic (eg, portal vein thrombosis), intrahepatic (eg, schistosomiasis), and posthepatic (eg, right-sided heart failure). ${ }^{1}$ In our patient, the dilated portal venules (inflow) in the presence of normal hepatic vein outflow suggested an increased intrahepatic resistance to blood flow. This finding excluded a causal role of the portal vein thrombosis and 

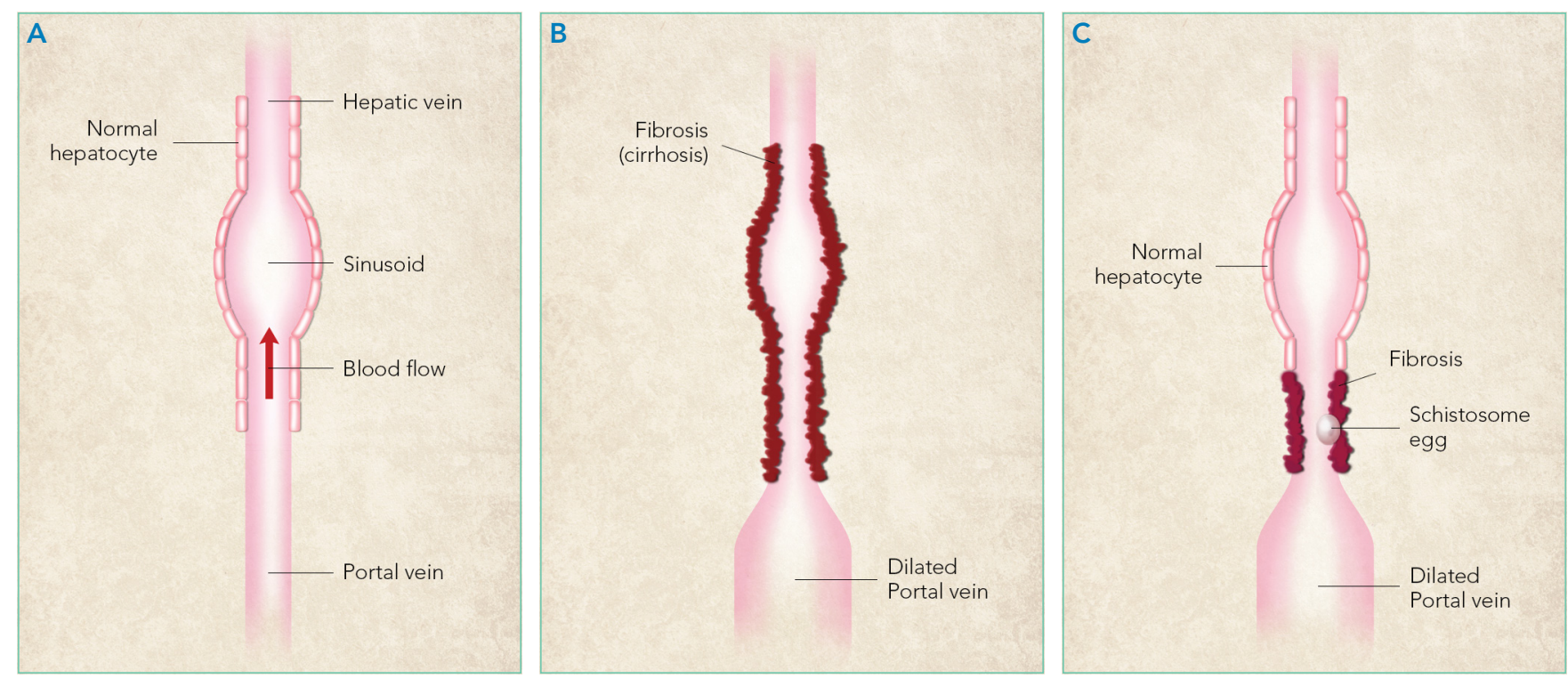

FIG 3. (A) In a normal liver, hepatocytes and adjacent connective tissue do not obstruct the blood flow of the portal venous system. (B) In cirrhosis, parenchymal fibrosis leads to loss of endothelial fenestrations and intrahepatic portal venous compression, which causes portal hypertension and proximal venous dilation. (C) In hepatic schistosomiasis, eggs that are lodged in the portal venous system cause granulomatous inflammation and portal venous fibrosis. The resultant obstruction to venous inflow leads to portal hypertension and proximal venous dilation. However, the hepatic architecture and function are usually maintained.

prompted testing for schistosomiasis.

Schistosomiasis affects more than 200 million people worldwide and is prevalent in Sub-Saharan Africa, South America, Egypt, China, and Southeast Asia. ${ }^{4,5}$ Transmission occurs in fresh water, where the infectious form of the parasite is released from snails. ${ }^{4,6}$ Schistosome worms are not found in the United States, but as a result of immigration and travel, more than 400,000 people in the United States are estimated to be infected. ${ }^{5}$

Chronic schistosomiasis develops from the host's granulomatous reaction to schistosome eggs whose location (depending on the species) leads to genitourinary, intestinal, hepatic, or rarely, neurologic disease. ${ }^{6}$ Hepatic schistosomiasis arises when eggs released in the portal venous system lodge in small portal venules and cause granulomatous inflammation, periportal fibrosis, and microvascular obstruction. ${ }^{6}$ The resultant portal hypertension develops insidiously, but the architecture and synthetic function of the liver is maintained until the very late stages of disease. ${ }^{6,7}$ Pulmonary hypertension can arise from the embolization of eggs to the pulmonary arterioles via portosystemic collaterals.

The demonstration of eggs in stool is the gold standard for the diagnosis of hepatic schistosomiasis, which is most commonly caused by Schistosoma mansoni and S. japonicum.? Serologic assays provide evidence of infection or exposure but may cross-react with other helminths. Liver biopsy may reveal characteristic histopathologic findings, including granulomatous inflammation, distorted vasculature, and the deposition of collagen deposits in the periportal space, leading to "pipestem fibrosis." 8,9 If eggs cannot be detected on stool or histology, then serology, secondary histologic changes, and sometimes PCR are used to diagnose hepatic schistosomiasis. In our patient, the epidemiology, Schistosoma antibody titer, pulmonary hypertension, and liver biopsy with granulomatous inflammation, periportal fibrosis, and intrahepatic portal venule dilation were diagnostic of hepatic schistosomiasis.

The recurrent episodes of confusion which resolved with lactulose therapy were suggestive of hepatic encephalopathy, which results from shunting and accumulation of neurotoxic substances that would otherwise undergo hepatic metabolism. ${ }^{10}$ Clinicians are most familiar with hepatic encephalopathy in cirrhosis, where multiple liver functions - synthesis, excretion, metabolism, and circulation - simultaneously fail. NCPH represents a scenario where only the circulation is impaired, but this is sufficient to cause the portosystemic shunting that leads to encephalopathy. Our patient's recurrent hepatic encephalopathy, despite adherence to lactulose and rifaximin and its resolution after praziquantel treatment, underscores the importance of addressing the underlying cause of portosystemic shunting.

Associating portal hypertension with cirrhosis is efficient and accurate in many cases. However, when specific manifestations of cirrhosis are lacking, clinicians must decouple this association and pursue an alternative explanation for portal hypertension. The presence of some intrahepatic pathology (from schistosomiasis) but no cirrhosis made this case a particularly tough egg to crack.

\section{TEACHING POINTS}

- In the developed world, $90 \%$ of portal hypertension is due to cirrhosis. Hepatic schistosomiasis is the most common cause of NCPH worldwide.

- Chronic schistosomiasis affects the gastrointestinal, hepatic, and genitourinary systems and causes significant global morbidity and mortality.

- Visualization of schistosome eggs is the diagnostic gold 
standard. Indirect testing such as schistosoma antibodies and secondary histologic changes may be required for the diagnosis in patients with a low burden of eggs.

Disclosures: Dr. Geha has no disclosures. Dr. Dhaliwal reports receiving honoraria from ISMIE Mutual Insurance Company and Physicians' Reciprocal Insurers. Dr. Peters' spouse is employed by Hoffman-La Roche. Dr. Manesh is supported by the Jeremiah A. Barondess Fellowship in the Clinical Transaction of the New York Academy of Medicine, in collaboration with the Accreditation Council for Graduate Medical Education (ACGME)

\section{References}

1. Sarin SK, Khanna R. Non-cirrhotic portal hypertension. Clin Liver Dis. 2014;18(2):451-76. doi: 10.1016/j.cld.2014.01.009.

2. Tapper EB, Lok AS. Use of liver imaging and biopsy in clinical practice. $N$
Engl J Med. 2017;377(8):756-768. doi: 10.1056/NEJMra1610570

3. Udell JA, Wang CS, Tinmouth J, et al. Does this patient with liver disease have cirrhosis? JAMA. 2012;307(8):832-42. doi: 10.1001/jama.2012.186.

4. Centers for Disease Control and Prevention. Parasites-Schistosomiasis. https://www.cdc.gov/parasites/schistosomiasis/. Accessed December 2, 2017.

5. Bica I, Hamer DH, Stadecker MJ. Hepatic schistosomiasis. Infect Dis Clin N Am. 2000;14(3):583-604.

6. Ross AG, Bartley PB, Sleigh AC, et al. Schistosomiasis. N Engl J Med. 2002;346(16):1212-20. doi: 10.1056/NEJMra012396

7. Gray DJ, Ross AG, Li YS, McManus DP. Diagnosis and management of schistosomiasis. BMJ. 2011;342: 2561-2561. doi: doi.org/10.1136/bmj.d2651.

8. Manzella A, Ohtomo K, Monzawa S, Lim JH. Schistosomiasis of the liver. Abdom Imaging. 2008;33(2):144-50. doi: 10.1007/s00261-007-9329-7.

9. Gryseels B, Polman K, Clerinx J, Kestens L. Human schistosomiasis. Lancet. 2006;368(9541):1106-18. doi: 10.1016/S0140-6736(06)69440-3.

10. Blei AT, Córdoba J. Practice Parameters Committee of the American College of Gastroenterology. Hepatic encephalopathy. Am J Gastroenterol. 2001;96(7):1968. doi: 10.1111/j.1572-0241.2001.03964.x. 